\title{
Liquid crystal cells based on photovoltaic substrates
}

\author{
L. Lucchetti \\ I.lucchetti@univpm.it \\ K. Kushnir
}

A. Zaltron

F. Simoni
Dipartimento SIMAU, Università Politecnica delle Marche, Ancona, Italy

Physics Faculty, Kyiv National Taras Shevchenko University, Prosp. Glushkova 2, Kiev, Ukraine

Dipartimento di Fisica e Astronomia G. Galilei, Università di Padova, via Marzolo 8, Padova, Italy

Liquid crystal cells with LiNb03:Fe crystals as substrates, are described. The photovoltaic field generated by the substrates is able to reorient the liquid crystal director thus giving rise to a phase shift on the light propagating through the cell, as in liquid crystal light valves. The process does not require the application of an external electric field, thus being potentially useful for applications requiring a high degree of compactness. An efficient optical switch with a high transmission contrast, based on the described optically-induced electric field, is also proposed.

[DOI: http://dx.doi.org/10.2971/jeos.2016.16007]

Keywords: Liquid crystals, nonlinear optics, lithium niobate

\section{INTRODUCTION}

It is known that the main mechanism of photorefraction in $\mathrm{LiNbO} 3$ crystals is the bulk photovoltaic effect $[1,2]$. In particular in iron-doped $\mathrm{LiNbO}_{3}\left(\mathrm{LiNbO}_{3}: \mathrm{Fe}\right)$ the photovoltaic effect is significantly enhanced due to the presence of two valence states of the iron dopant: $\mathrm{Fe}^{2+}$ (donor ions) and $\mathrm{Fe}^{3+}$ (acceptor ions). Such an effect consists in the appearance of a photoinduced current, whose corresponding electric field is called photovoltaic field, generated upon illumination according to the scheme: $\mathrm{Fe}^{2+}+h v \longleftrightarrow \mathrm{Fe}^{3+}+\mathrm{e}^{-}$. The subsequent charge distribution which takes place inside the crystal gives rise to an electric field with saturation values that can reach $10^{5} \mathrm{~V} / \mathrm{cm}$, depending on iron concentration and on the reduction degree (i.e. the ratio between $\mathrm{Fe}^{2+}$ and $\mathrm{Fe}^{3+}$ ions) of the material [1].

If two $\mathrm{LiNbO}_{3}: \mathrm{Fe}$ crystals of this kind are used as substrates in a liquid crystal (LC) cell in the proper configuration, the charge distributions caused by the photoinduced current in each substrate give rise to a field between the two substrates. Such optically-generated electric field induces a rotation of the LC director with a consequent phase shift of the propagating light, thus giving rise to a nonlinear optical effect that is to a feedback action on the light itself due to its effect on the material. Due to the absence of electric contacts for the application of the external electric field, a similar effect could be useful for several applications. For instance in the realization of Optically Addressed compact Spatial Light Modulators, Liquid Crystal Light Valves or for optical devices to be integrated in very small platforms such as those used in microfluidic systems.
We should mention that a similar kind of cell has been proposed by D. R. Evans et al. in 2006 [3], where the onset of the photovoltaic effect was reported. In that paper the authors discuss the transmission properties of such a cell as due to a superposition between the photovoltaic effect and the two beam coupling originated by the photorefractive properties of the crystals. However data concerning the phase shift induced on the liquid crystalline material are missing.

Here we report the direct evidence of LC reorientation under the optically-generated electric field and measure the induced phase shift. We show that by using a LC cell with $z$-cut $\mathrm{LiNbO}_{3}: \mathrm{Fe}$ crystal substrates and a single unfocused pump beam for homogeneous illumination, a light-induced control of the phase shift can be easily achieved in a time range of few seconds, corresponding to an induced optical anisotropy $\Delta n$ of about 0.1 , making negligible any contribution originated by photorefractive nonlinearities of the $\mathrm{LiNbO}_{3}: \mathrm{Fe}$ crystals. An example of possible application is also described.

\section{EXPERIMENTAL DETAILS}

The LC cell we propose is obtained by using two $z$-cut $\mathrm{LiNbO}_{3}: \mathrm{Fe}$ thin crystals $(900 \mu \mathrm{m})$ as substrates. The crystals were obtained by a boule of lithium niobate doped with iron $\left(c_{\mathrm{Fe}}=18.9 \cdot 10^{18} \mathrm{~cm}^{-3}=0.1 \% \mathrm{~mol}\right)$ grown at the University of Padua by using the Czochralski technique. The growth direction was along the $z$-axis (that corresponds to the $c$-axis) of the material, with a pulling rate of $2 \mathrm{~mm} / \mathrm{h}$. After the growth, the 


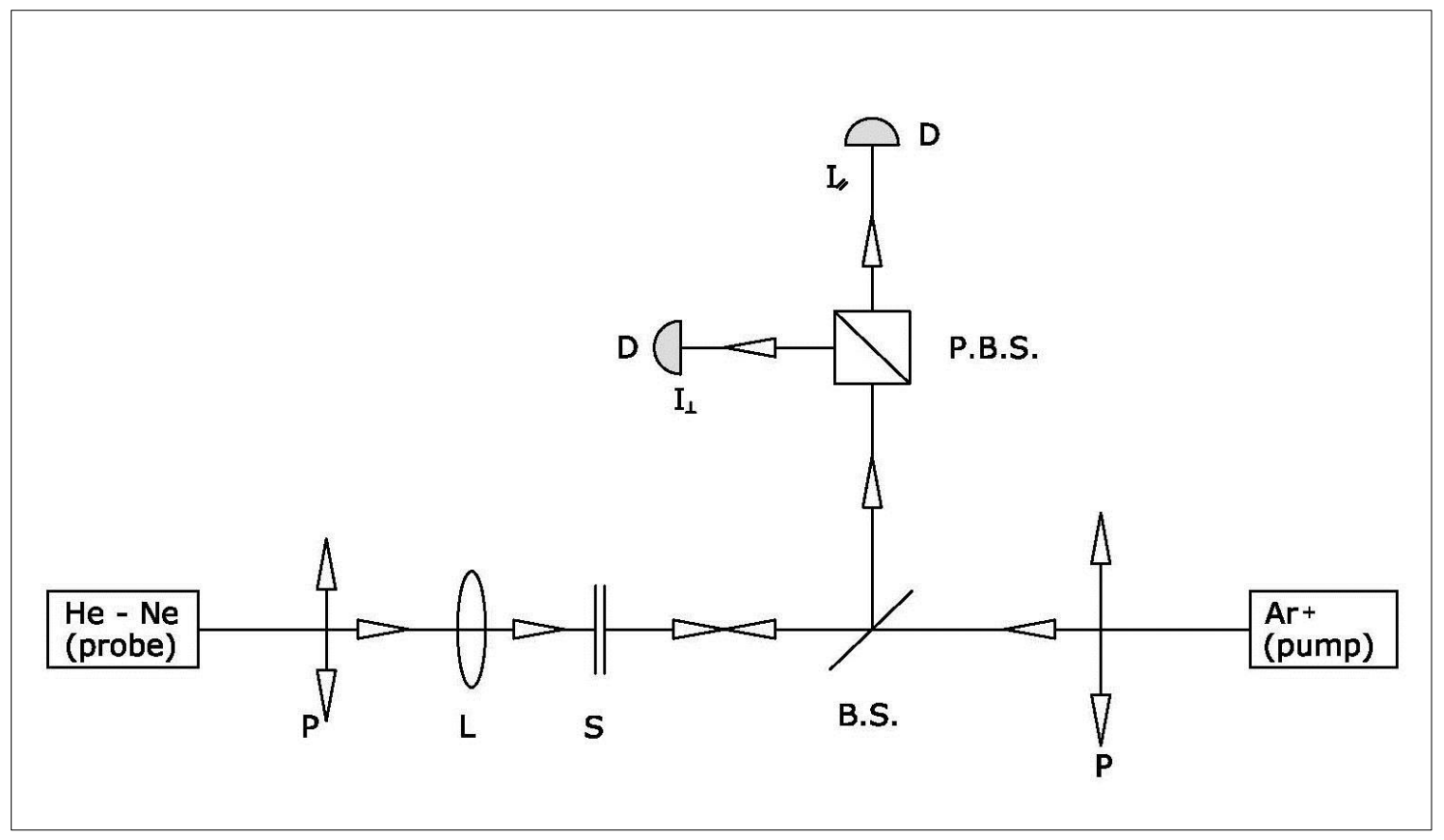

FIG. 1 Experimental set-up. P: polarisers, L: lens, S: sample, B.S.: beam splitter, P.B.S.: polarizing beam splitter, D: detectors. Pump beam has a linear polarization parallel to the rubbing direction; probe is linearly polarized in order to form an angle of $45^{\circ}$ with this latter.

$\mathrm{LiNbO}_{3}: \mathrm{Fe}$ boule was poled in air atmosphere at $1200^{\circ} \mathrm{C}$ in order to obtain a single domain crystal. By means of High Resolution X-Ray Diffraction measurements the crystalline quality of the material was checked to be compatible with commercial crystals and the boule was oriented along the three main crystallographic axes. The main faces of the samples are perpendicular to the $z$-axis, so that it is possible to exploit the photoinduced current and the consequent charge accumulation which arises along this axis under illumination. The reduction degree is about $0.02 \pm 0.01$. The two $\mathrm{LiNbO}_{3}: \mathrm{Fe}$ crystals were rubbed with a cotton tissue before cell assembling, in order to obtain planar alignment of the LC molecules. Cell thickness, controlled by mylar spacers, was 6 and $13 \mu \mathrm{m}$. The used LC is the nematic penthylcianobiphenil 5CB (from Nematel) inserted in the cell by capillarity. The good planar alignment of the final samples was checked under polarizing optical microscope.

Cells were irradiated by the green line of an Ar+ laser $(\lambda=514 \mathrm{~nm})$ at normal incidence. The pump beam has a linear polarization parallel to the rubbing direction. In this configuration light propagates along the $c$-axis of both $\mathrm{LiNbO}_{3}: \mathrm{Fe}$ crystals and it does not undergo any phase shift due to the crystals birefringence. Pump power is fixed at $177 \mathrm{~mW}$, corresponding to an intensity of $10 \mathrm{~W} / \mathrm{cm}^{2}$. It is worth noting that, due to the low reduction degree of the used substrates, the temperature rise in iron-doped $\mathrm{LiNbO}_{3}$ crystals is expected to be very low with this intensity in the used time scale. Temperature increase of some tenths of $\mathrm{K}$ has been observed for reduction degrees one or two orders of magnitude higher and for irradiation times on the order of several minutes, whereas the maximum exposure time used here is $10 \mathrm{~s}$.

As mentioned, light irradiation produces an electric field in the region filled by the LC. The effect of the light-induced electric field on the LC is to rotate the director thus producing a smooth transition from the planar to the homeotropic configuration. This director rotation produces on its turn a phase shift on the light propagating through the cell, that can be measured with a pump-probe technique, as described in [4]. The used probe beam comes from a low power He-Ne laser $(\lambda=633 \mathrm{~nm})$, is counter propagating with respect to the green pump beam and is linearly polarized in order to form an angle of $45^{\circ}$ with the cell rubbing direction. This beam is focused on the sample at the center of the pump spot. The probe light transmitted by the sample is divided by a polarizing beam splitter into two beams: one polarized parallel, the other perpendicular to the first polarizer placed before the sample. A sketch of the experimental set up is shown in Figure 1.

\section{RESULTS AND DISCUSSION}

The light-induced phase shift $\Delta \phi$ is determined from the ratio between the intensities $I_{\perp}$ and $I_{\|}$of the two transmitted beams, according to the formula [4]:

$$
\begin{array}{cc}
\Delta \phi=N \pi+2 \tan ^{-1} \frac{I_{\perp}}{I_{\|}} & \text {if } N=0,2,4, \ldots \\
\Delta \phi=(N+1) \pi+2 \tan ^{-1} \frac{I_{\perp}}{I_{\|}} & \text {if } N=1,3,5, \ldots
\end{array}
$$

where the integer $N$ is the number of extrema (maxima and minima) in the curves $I_{\perp}$ and $I_{\|}$(see the inset of Figure 2). The two signals have been detected as a function of the exposure time.

Results show very clearly that LC director actually changes orientation under light irradiation. A detailed analysis of the induced phase shift as a function of time and for different cell thicknesses has been carried on. As an example, Figure 2 shows $\Delta \phi$ versus $t$ for a $13 \mu \mathrm{m}$ thick cell. Thinner cells exhibit a lower maximum phase shift, as expected. The inset reports the behavior of the probe transmitted light for the two 


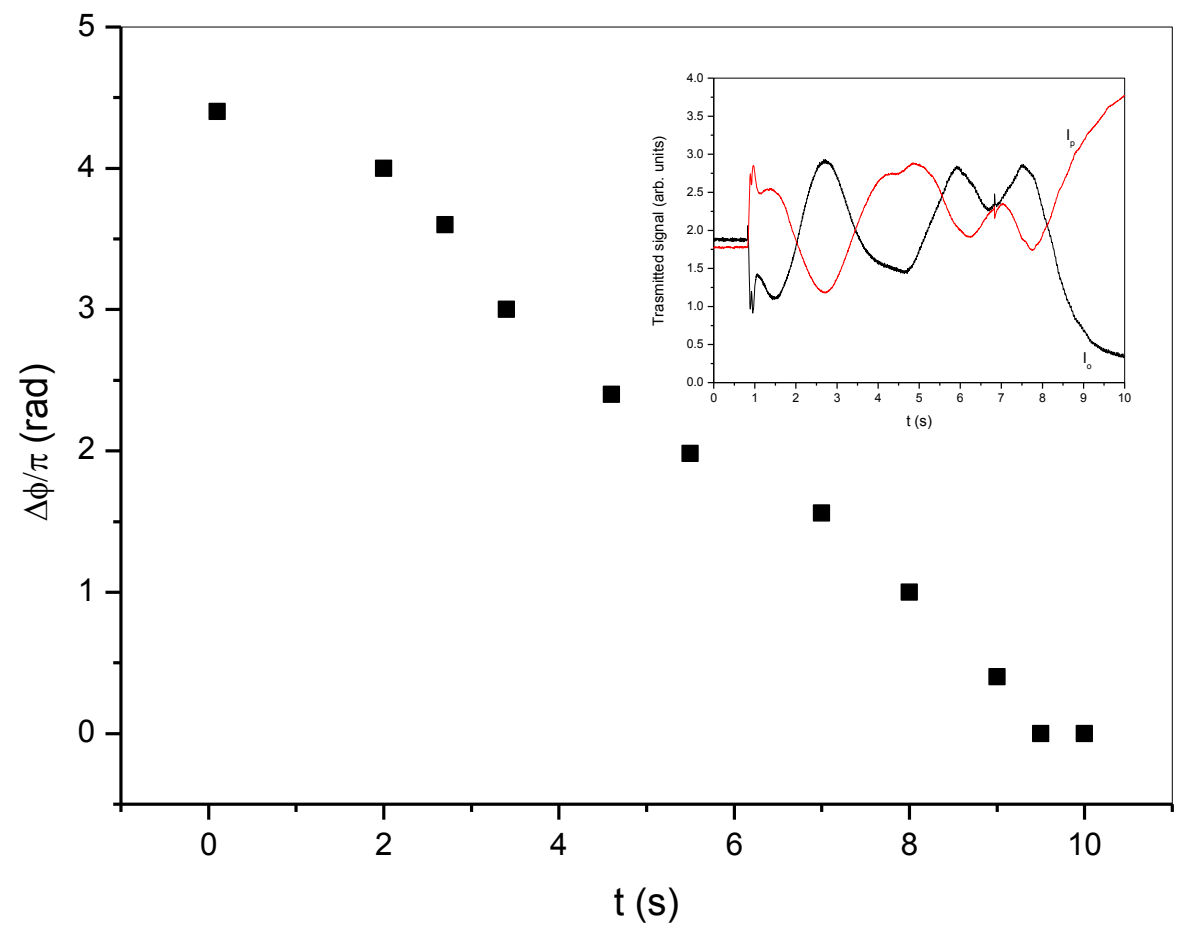

FIG. 2 Induced phase shift as a function of the irradiation time. Inset: transmitted probe light with parallel $\left(I_{\|}\right)$and orthogonal $\left(I_{\perp}\right)$ polarization with respect to the probe beam incident on the cell.

detected polarizations: parallel and orthogonal to the one incident on the cell. The signals show the typical oscillations observed when a planar sample, in the same geometry as the one used in the experiment, is submitted to an increasing voltage, where each additional peak corresponds to an increase of $\pi$ of the phase shift between the two waves. The final situation corresponds to almost uniform director alignment perpendicular to the substrates, thus allowing transmission of the signal parallel to the incoming polarization. It is worth remarking that the curves shown in the inset of Figure 2 cannot be as symmetric and "clean" as those obtained in the classical measurement of the phase shift in a liquid crystal conventional cell, where the transmitted signal is detected versus the externally applied voltage. Here the transmitted signal is detected as a function of the exposure time and the shape of the oscillations is affected by the relationship between this parameter and the photovoltaic voltage across the cell. Also the weak contrast between the two signals can be reasonably ascribed to the same reason.

Similar measurements have been carried out both on "ordinary" cells constructed with glass substrates and on empty cells made only by the two crystal substrates. In both cases the resulting signals were flat ruling out any contribution from the direct effect of light on the LC and demonstrating that any nonlinear response originated by photorefraction occurring inside the lithium-niobate is negligible in the used time scale.

The shape of the induced phase shift reported in Figure 2 is very similar to the one typical of the liquid crystal light valves [5]. Note that the value of the maximum phase shift is strictly dependent on the quality of LC alignment, thus a further optimization of the cell preparation is expected to produce higher values of $\Delta \phi$. It is well known that the induced phase shift is related to the induced optical anisotropy $\Delta n$ through the relation [6] $\Delta \phi=(2 \pi / \lambda) d \Delta n$. From data of Figure 2 one gets a maximum index modulation $\Delta n_{\max } \cong 0.1$, that is higher than the one achievable with a phototrefractive crystal $[7,8]$ thus confirming the main role played by LC director reorientation induced by the photovoltaic field.

The possibility of applying an external electric field to a LC cell optically, without using external contacts, can in principle open the way to a number of interesting applications. As an example, we propose an optical switch obtained by using the same pump probe configuration described above. The probe beam is again polarized at 450 with respect to the rubbing direction and the red light transmitted by the cell is polarized orthogonally to the incident one. Due to the initial planar configuration of the cell, this situation corresponds to maximum transmittance. When the cell is irradiated with the pump beam, the transmittance of red light decreases to a minimum value in times depending on the LC rotational viscosity and dielectric anisotropy and on the value of the electric field generated by the $\mathrm{LiNbO}_{3}: \mathrm{Fe}$ substrates during light irradiation. Figure 3 shows the probe transmitted light during pump irradiation of a $13 \mu \mathrm{m}$ thick cell. The exposure time is $1 \mathrm{~s}$ in Figure 3(a) and $2 \mathrm{~s}$ in Figure 3(b). It can be seen that the contrast ratio between on/off states increases with irradiation time, reaching $80 \%$ for $2 \mathrm{~s}$ irradiation. However, longer exposure would lead to the onset of the signal oscillations observed in the inset of Figure 2. Two seconds seems thus the optimum irradiation time for a good optical switch.

The switch on and switch off times are of the order of $1 \mathrm{~s}$ in both cases. Faster switch on times have been measured in other cells, the minimum observed value being $50 \mathrm{~ms}$ for a $6 \mu \mathrm{m}$ cell, where, however, the difference between maximum and minimum transmittance was lower than that reported in 


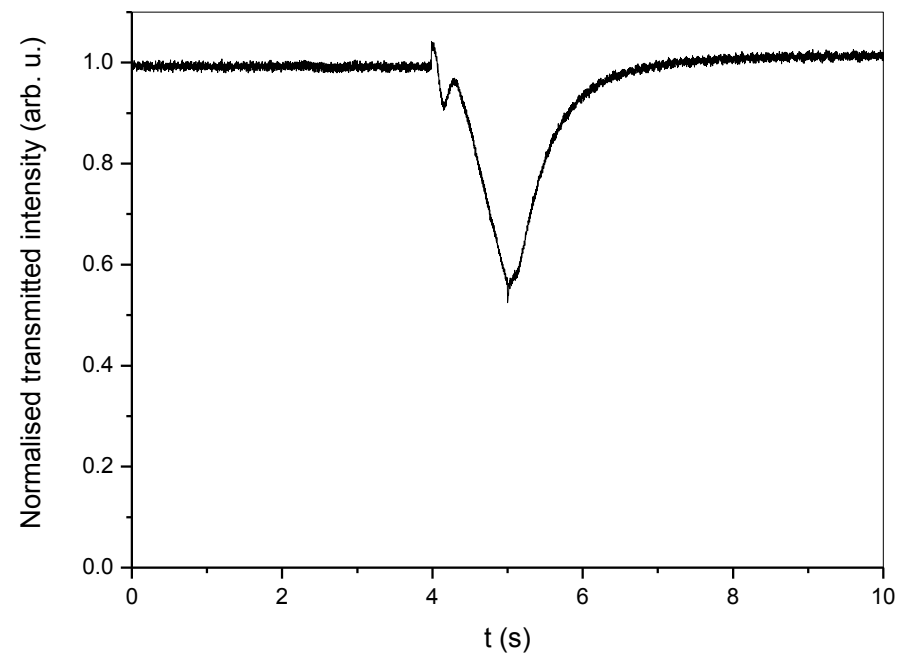

(a)

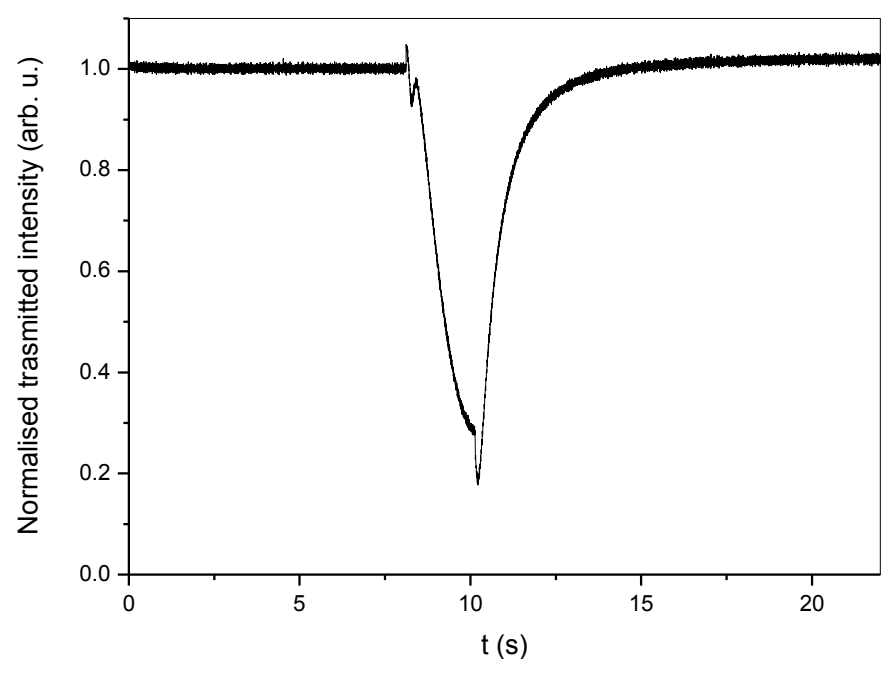

(b)

FIG. 3 Transmitted probe intensity under $1 \mathrm{~s}$ (a) and $2 \mathrm{~s}$ (b) pump irradiation. Cell thickness: $13 \mu \mathrm{m}$.

Figure 3(b). The reason for a so wide distribution of switch on times is not clear yet, even if small differences in the reduction degree, present in the used $\mathrm{LiNbO}_{3}: \mathrm{Fe}$ substrates, can affect the crystal response and change to a certain extent the behavior of different cells. However, the observation of response times down to $50 \mathrm{~ms}$, demonstrates the possibility of improving the speed of the obtained optical switch.

The parameters governing the switch off time are fixed by the kind of LC used (fixing the sample temperature), according to the relation [6]:

$$
\tau_{\text {off }} \cong \frac{\gamma d^{2}}{K \pi^{2}}
$$

where $\gamma, d$ and $K$ are the LC rotational viscosity, the cell thickness and the elastic constant, respectively. On the contrary, those affecting the switch on time follow the relation [9]:

$$
\tau_{\mathrm{on}} \cong \frac{\gamma}{\epsilon_{0}|\Delta \epsilon| E^{2}}
$$

$\epsilon_{0}$ and $\Delta \epsilon$ being the dielectric constant and the dielectric anisotropy of the LC and E the electric field created in the cell by the two photovoltaic substrates. This latter is not fixed by the particular kind of LC used and can be tailored a little bit.
In principle it can be increased by acting on the dopant concentration of the substrates and on the reduction degree, but at the expense of the maximum cell transmission. Moreover, a higher pump intensity with respect to that used in the present work, should lead to a square dependence of the photovoltaic current on the intensity $I$ itself [10] thus to the possibility of enhancing the photovoltaic field by increasing $I$.

\section{CONCLUSIONS}

In conclusion we reported on LC cells made with two photovoltaic crystals as substrates. The photovoltaic current created in the crystals upon light illumination, gives rise to an electric field between the substrates able to reorient the LC director as an externally applied electric field does. The induced reorientation has been directly proved by a pump-probe technique and a consequent phase shift of few $\pi$ s has been measured. A possible application of the proposed system as optical switch has also been proposed.

\section{References}

[1] T. Volk, and M. Wohlecke, Lithium niobate (Springer-Verlag, Berlin/Heidelberg, 2008).

[2] K. Buse, "Light-induced charge transport processes in photorefractive crystals I: models and experimental methods," Appl. Phys. B 64, 273-291 (1997).

[3] J. L. Carns, G. Cook, M. A. Saleh, S. V. Serak, N. Tabiryan, and D. R. Evans, "Self-activated liquid crystal cells with photovoltaic substrates," Opt. Lett. 31, 993-995 (2006).

[4] S.-T. Wu, U. Efron, and L. D. Hess, "Birefringence measurements of liquid crystals," Appl. Opt. 23, 3911-3915 (1984).

[5] S. Residori, U. Bortolozzo, and J. P. Huignard, "Adaptive holography in liquid crystal light-valves," Materials 5, 1546-1559 (2012).

[6] F. Simoni, Nonlinear optical properties of liquid crystals and polymer dispersed liquid crystals (World Scientific, Singapore, 1997).

[7] G. Bettella, G. Pozza, A. Zaltron, M. V. Ciampolillo, N. Argiolas, C. Sada, M. Chauvet, et al.,"Integrated opto-microfluidics platforms in lithium niobate crystals for sensing applications," Proc. SPIE 9365, 936517 (2015).

[8] A. M. Glass, D. von der Linde, and T. J. Negran, "High-voltage bulk photovoltaic effect and the photorefractive process in $\mathrm{LiNbO}_{3}$, Appl. Phys. Lett. 25, 233-235 (1974).

[9] V. Borshch, S. V. Shiyanovskii, and 0. D. Lavrentovich, "Nanosecond electro-optic switching of a liquid crystal," Phys. Rev. Lett. 111, 107802 (2013).

[10] M. Simon, S. Wevering, K. Buse, and E. Kratzig, "The bulk photovoltaic effect of photorefractive $\mathrm{LiNbO}_{3}$ :Fe crystals at high light intensity," J. Phys. D Appl. Phys. 30, 144-149 (1997). 